\title{
SCALING PROPERTIES OF EPIDEMIOLOGICAL TIME SERIES
}

\author{
A. HARNOS ${ }^{1}$ - J. REICZIGEL ${ }^{1}-$ F. RUBEL ${ }^{2}-$ N. SOLYMOSI ${ }^{1}$ \\ ${ }^{1}$ Department of Biomathematics and Informatics, Faculty of Veterinary Science, \\ Szent István University, \\ H-1078, István u. 2. Budapest, Hungary \\ (phone: +36-1-478-4214; fax: +36-1-478-4217) \\ ${ }^{2}$ Biometeorology and Mathematical Epidemiology Group Department of Natural Sciences, \\ University of Veterinary Medicine Vienna \\ Veterinärplatz 1, A-1210 Vienna, Austria \\ *e-mail: Harnos.Andrea@aotk.szie.hu \\ (Received $10^{\text {th }}$ Sep 2005, accepted $10^{\text {th }}$ Oct 2006)
}

\begin{abstract}
The statistical analysis monthly Hungarian (1967-2001) and Austrian (1989-2003) rabies and Hungarian bovine tuberculosis (1950-1979) cases were statistically analyzed. There were eradication campaigns against both diseases, which gave us the unique opportunity to observe how the statistical properties of the time series changed due to the eradication program. The fluctuations around the trend were got by removing the 12 month moving averages from the time series.

In order to characterize the structure of the contacts among the individuals exposed to the diseases and the underlying processes governing the behavior of the epidemics, the fluctuations were analyzed before and after eradication separately. It turned out that the tails of the complementary cumulative distribution functions differ in the rabies cases, and do not differ in the case of tuberculosis. In each case the tail of the distribution follows an Inverse Power Law (IPL) function and describes the distribution of extreme events. It is possible to make conclusions about the dimensions of the processes from the fitted exponents. These dimensions are not related necessarily to the spatial dimensions, but to the possible connections. The knowledge about the distributions give us the opportunity to asses the risk of epidemic outbreaks.

Keywords: epidemiology, immunization, rabies, time series analysis, IPL distribution
\end{abstract}

\section{Introduction}

Instead of using the usual statistical models, Rhodes and Anderson [1][2] [3], [4] proposed a new stochastic model, the so called Invers Power Law function (IPL), or otherwise Pareto model for describing time series of the epidemic sizes from small and large vaccinated human populations. Trottier and Philippe [5] applied this model for measles, rubella, pertussis and mumps outbreaks in Canada. IPL is a function with no characteristic scale and self-similar upon rescaling (scale invariance), and it may fit to the extreme values of the distribution (power law tail). The IPL function is self-similar against rescaling (scale invariance), that means that several scales are hiararchically embedded in each other, and this feature can explain the co-existence of small and very large epidemics. Large outbreaks are expected from this type of distributions [6]. One possible explanation of the existence of the IPL interactions assume that the system of the contacts among the individuals (contact network) is not random (Erdős-Rényi type). In scale-free networks, a few node have several connections (supernodes), but for the most only a few have (Fig. 1).

One can draw conclusions about the topolgy of the contact network where the epidemic spread from the distribution of the epidemic sizes [5] or can reveal the 
underlying mechanisms [7]. The interactive network of the contacts can explain the temporal and spatial pattern of the epidemic.


Figure 1. Exponencial and scale invariant contact network

Our goal is to prove the hypothesis that like in the case of human epidemics [5], in the cases under investigation, the distribution of the epidemic sizes of animals follows also an IPL function.

\section{Modeling}

The method of fitting an IPL function is called scaling analysis [1][2][3] and it consists of fitting the $F_{c}(l)=a l^{b}$ function to the epidemic size distribution via fitting a linear function on the $\operatorname{logarithmic}$ scale $\log F_{c}(l)=\log a+b \log l$, where 1 is the epidemic size (in our case normalized with the moving average), a and $b$ (a negative number) are estimable parameters of the epidemic size distribution.

\section{The epidemics}

\section{Rabies}

Rabies is a lethal viral zoonosis that can spread to humans and other animals through biting. During the last century, rabies has spread throughout parts of Central and Western Europe. Foxes have been the main hosts, but many other animals have also been infected, particularly dogs and cats. The incidence of endemic, fox-adapted rabies in Central and Western Europe has fallen dramatically. This has been largely due to the vaccination of wild and domestic animals.

In Austria and Hungary only the urban type of rabies - spread by dogs - existed until 1954. The sylvatic type rabies - spread by foxes - appeared in 1954 coming from the North-East. The epidemic propagated at a speed of 50-60 km/year until rabies became an endemic disease in 1970 [8].

In 1992, an immunization campaign started in both countries. 
In Hungary the oral vaccination campaign of the red foxes had several steps. In 1996 the whole western part was covered by the program. The eastern part of the country was not eradicated until 2001.

It is convenient to handle separately the western and eastern parts of Hungary because they are separated by the river Danube, a natural barrier for the spread of infection in Hungary, as it is quite unlikely for the foxes to pass through.

\section{Bovine tuberculosis}

Bovine tuberculosis is a significant zoonosis that can spread to humans through aerosols and by ingestion of raw milk. In developed countries, eradication efforts have significantly reduced the prevalence of this disease, but reservoirs in wildlife make complete eradication difficult. Bovine tuberculosis is still common in less developed countries, and economic losses can occur in cattle from deaths, chronic disease, and trade restrictions. Infections may also be a serious threat to endangered species [9][11]

Bovine tuberculosis results from infection by Mycobacterium bovis, a Gram positive, acid-fast bacterium.

In Hungary the eradication program against Bovine tuberculosis started in 1962 and it was finished by 1980 .

\section{Materials and methods}

Data

The Hungarian rabies data have been collected from the rabies case registry of the Animal Health and Food Control Department of the Hungarian Ministry of Agriculture. The resulting data base contains data of all documented rabies cases for the period of 1990-2001, including the location and date of occurrences as well as the species affected. In addition to this detailed data base, we have monthly count data for the period of 1967-1990 for every county.

The bovine tuberculosis data also have been collected from the Animal Health and Food Control Department of the Hungarian Ministry of Agriculture. In this case we have half monthly count data for the whole country (1950-1978).

The Austrian data have been got from the Bundesanastalt für Tierseuchenbekämpfung in Mödling and the data were processed by the Biometeorology and Mathematical Epidemiology Group Department of Natural Sciences, University of Veterinary Medicine Vienna.

We constructed monthly summarized time series from the Hungarian rabies dataset (1967-2001) for the western (Transdanubia), and for the eastern part (Eastern Hungary) of the country. In Fig. 2 we show these two time series. 


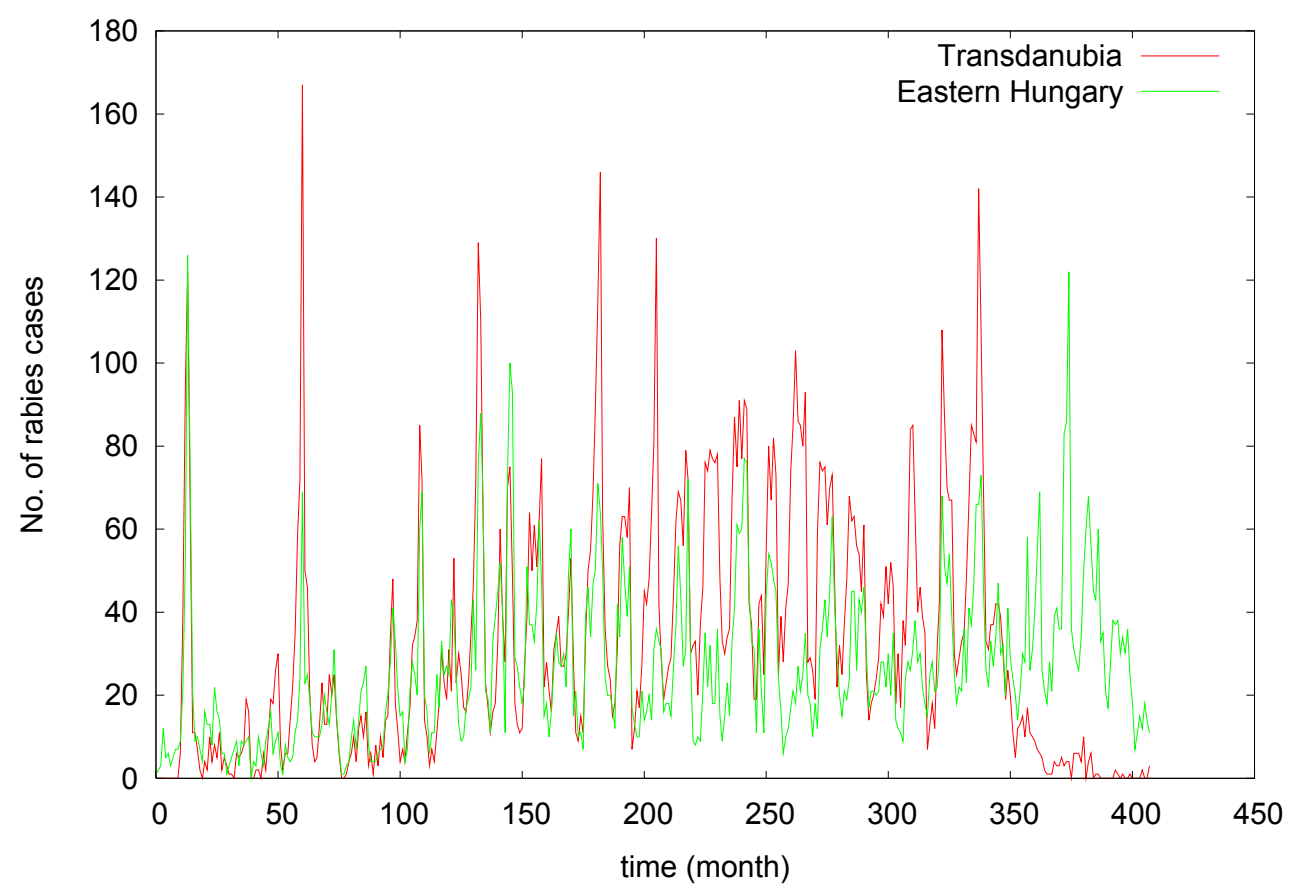

Figure 2. Monthly number of rabies cases in Transdanubia and in Eastern Hungary $(1967-2001)$

In the Austrian case we have summarized count data for the whole country (19892001). The constructed time series can be seen on Fig. 3.

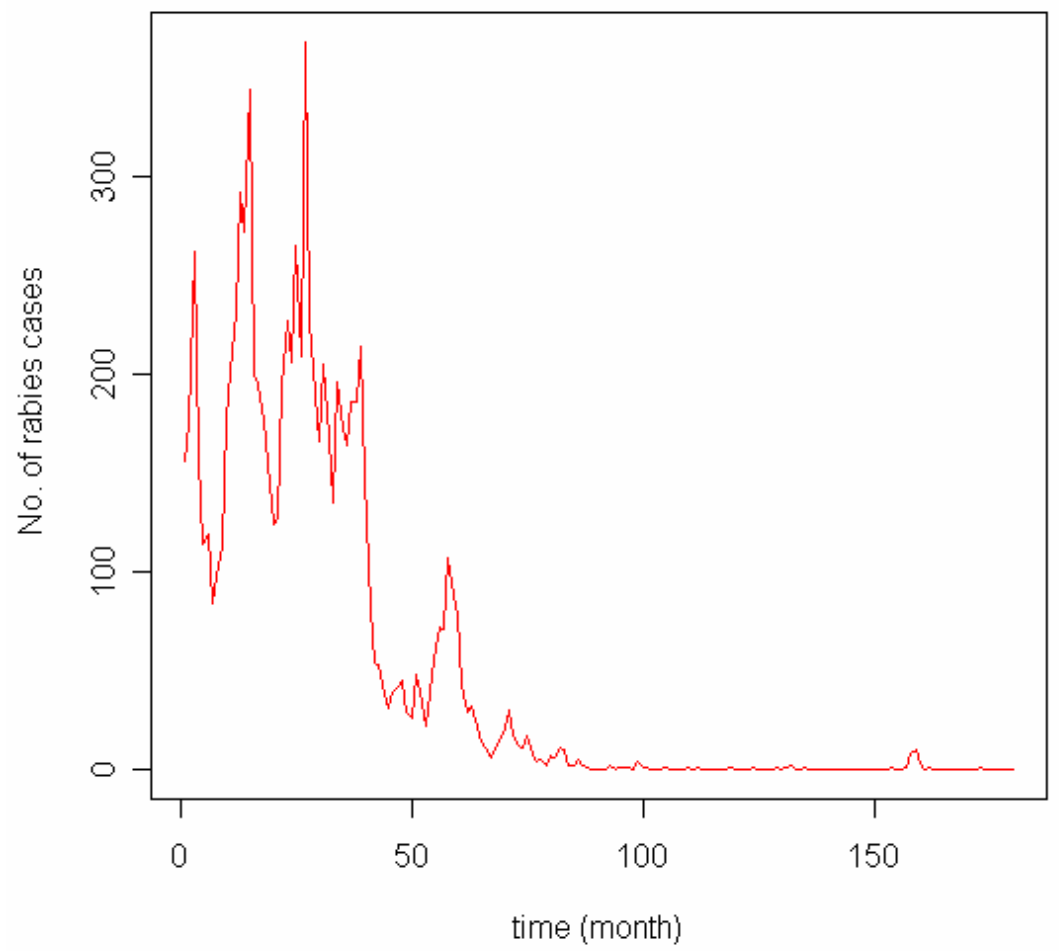

Figure 3. Monthly number of rabies cases in Austria (1989-2001) 
In the case of bovine tuberculosis Fig. 4 shows the time series.

Bovine tuberculosis time series (1950-1978)

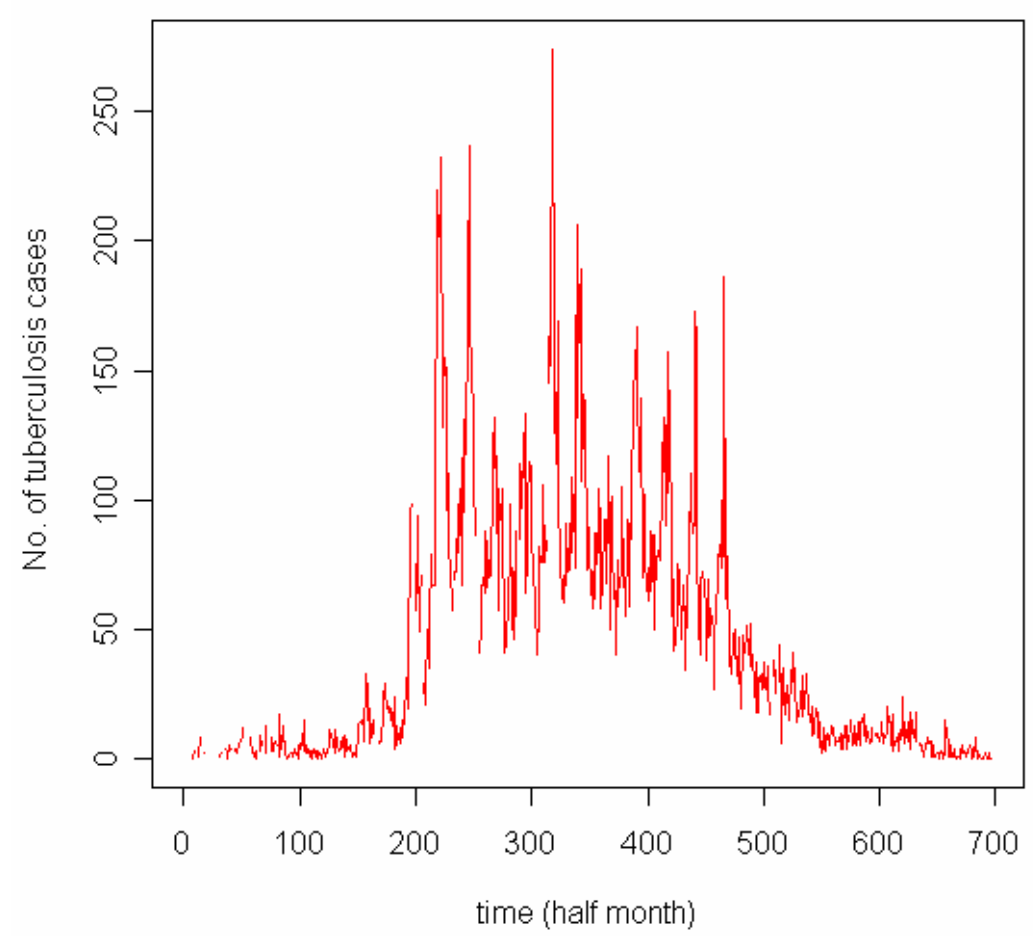

Figure 4. Half-monthly number of tuberculosis cases (1950-1978)

\section{Software}

The data base was developed using the Microsoft Office XP Professional Edition Microsoft Access software. We performed the analysis with Microsoft Excel XP, R and Gnuplot programs.

\section{Analysis of extreme fluctuations}

To analyze the large fluctuations, first we had to identify the trend in the time series. To calculate the moving averages as the trend is a good method if the average of the time series changes rapidly. In such cases it is useful to calculate the fluctuations around the moving average over a preceding period. In our case the external conditions have a 12-month periodicity, so it is natural to consider a 12-month moving average and values relative to the moving average $l(i)=f(i) /\left(\sum_{j=1}^{12} f(i-j) / 12\right)$, where $f(i)$ is the number of cases in the $i^{\text {th }}$ month in the series. These are the fluctuations, that we analyse further. It turned out that in each case the fluctuation time series are stationary.

The best way to study the statistics of the extremely large outbreaks is to consider the complementary probability distribution $F_{c}(l)=\operatorname{Pr} o b\{l(i)>l\}$, which gives the probability that the relative fluctuation is larger than $l$. By a scaling analysis - fitting 
lines to the tails on double logarithmic plots - it can be shown that this distribution develops an inverse power law tail

In Fig. 5-7 we show the complementary cumulative distribution for the data series with and without the eradicated parts for rabies in Transdanubia and Austria and for tuberculosis.

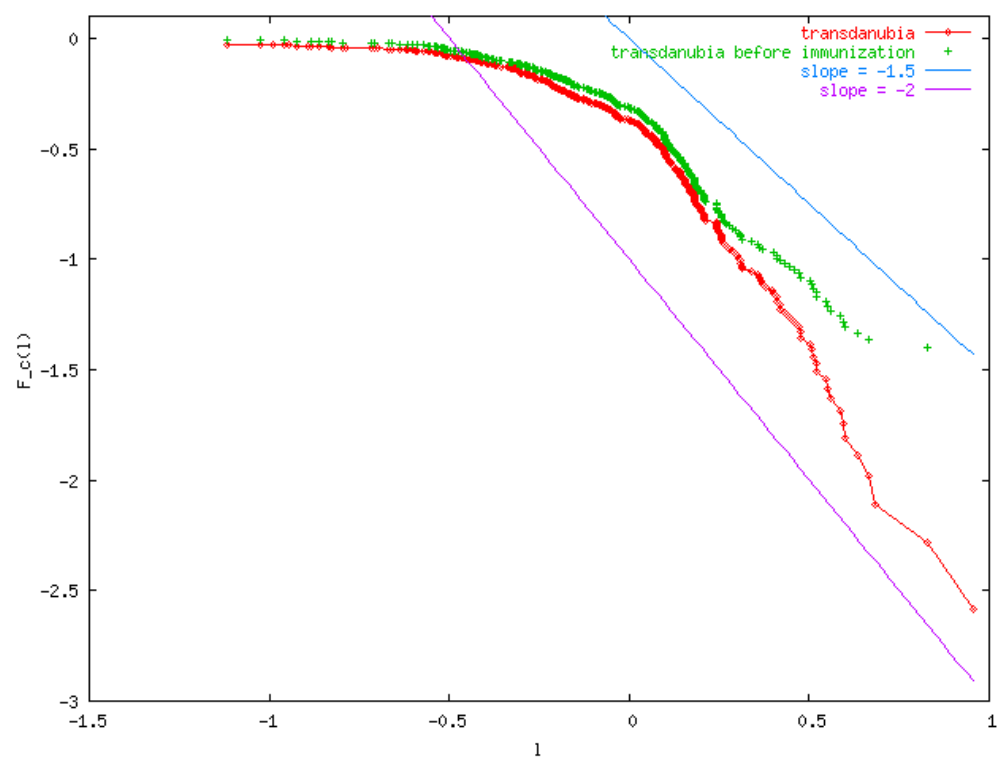

Figure 5. The complementary cumulative distribution of the fluctuations relative to the 12 months moving average for the Transdanubia data with and without immunization on a doubly logarithmic plot. The straight lines represent the fitted power law tails with exponent $b=-2$ and $b=-1.5$..

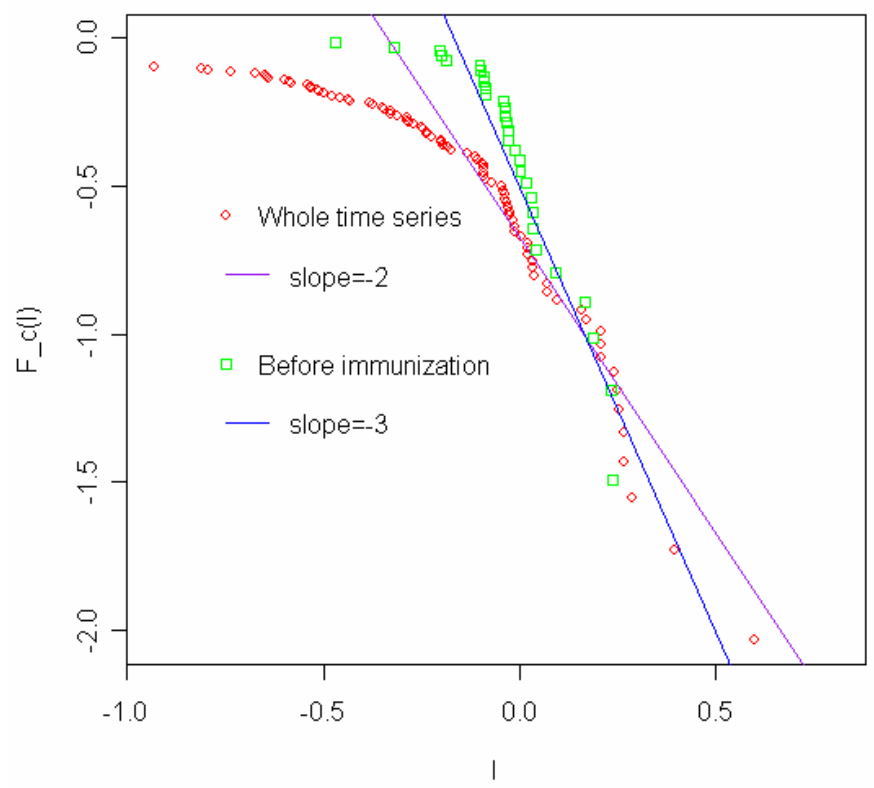

Figure 6. The complementary cumulative distribution of the fluctuations relative to the 12 months moving average for the Austrian data with and without immunization on a doubly logarithmic plot. The straight lines represent the fitted power law tails with exponent $b=-2$ and $b=-3$. 


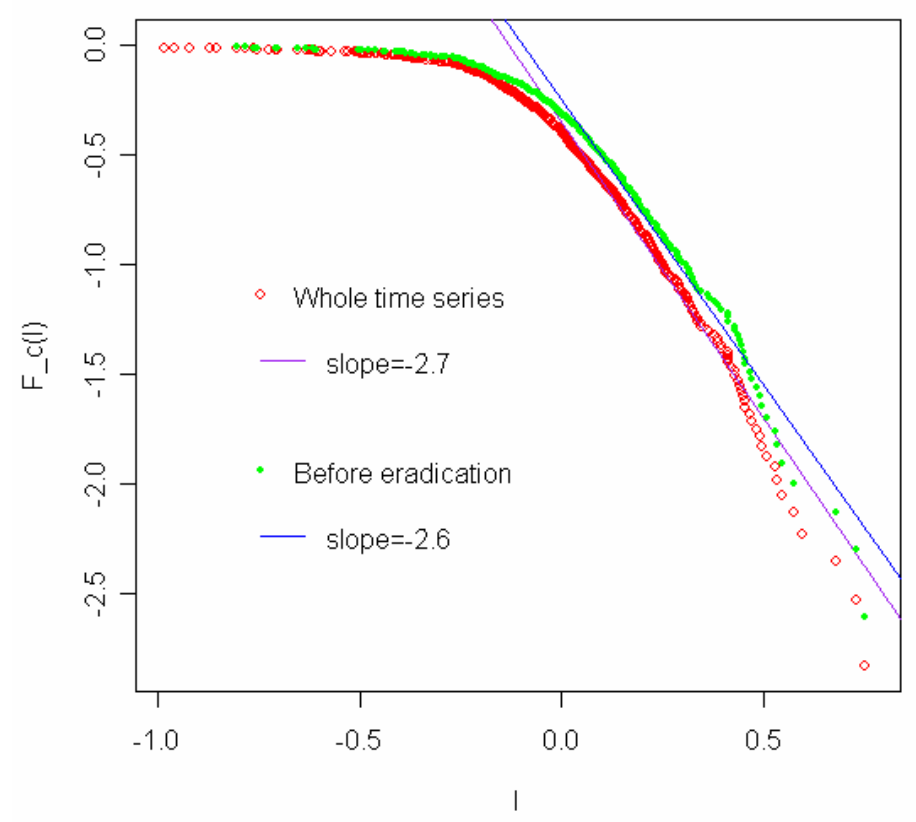

Figure 7. The complementary cumulative distribution of the fluctuations relative to the 12 months moving average for the tuberculosis data with and without eradication on a doubly logarithmic plot. The straight lines represent the fitted power law tails with exponent $b=-2.7$ and $b=-2.6$

In the Transdanubia case the main effect of immunization on the statistics of large outbreaks is the change of the scaling exponent $b$ of the IPL from about -1.5 before immunization to 2.0 after immunization.

In the austrian case the main effect of immunization on the statistics of large outbreaks is the change of the scaling exponent $b$ of the IPL from about -3 before immunization to -2 after immunization.

In the tuberculosis case there can not be seen significant change in the slopes.

\section{Discussion}

Rabies has always been given due respect both in human and veterinary medicine because it is an incurable disease. Most rabies cases in Europe are still diagnosed in red foxes (Vulpes vulpes) because red fox is the reservoir and primary perpetrator of the disease.

Bovine tuberculosis is also a zoonosis that can infect humans seriously.

In this paper we concentrated on extreme fluctuations in the time series constructed by calculating the fluctuations around the trends. Extreme fluctuations are lowprobability, high-consequence events [12] representing the majority of total losses. Understanding the size distribution of extreme events makes it possible to assess the risks of outbreaks.

We determined the distributions of extreme fluctuations which is a scale invariant power law distribution for the time series investigated.

Interesting to see that in the rabies cases the exponents (slopes on double logarithmic plots) definitely changed due to the eradication, this is different in the case of bovine 
tuberculosis. Possible explanation is that the contact network of the population did not change in this case, because cattles are domestic animals, and the location of the settlements where they are kept remained the same.

Due to the fact that the exponent of the power law is related to the dimensionality of the process [7] - that is not by all means spatial dimension - we could show how immunization changed the structure of the problem.

The importance of the IPL distribution of the extreme fluctuations (outbreaks of the epidemics) is that from this kind of epidemic spreading contact networks large outbreaks are expectable. As the pathogens of the diseases still exsist in the populations on a very low level, these pathogens may couse serious outbreaks in the future.

This kind of analysis can help to estimate the effects of eradication programs and to asses the risk of epidemic outbreaks.

Acknowledgements: This work was partly supported by the grants OTKA T049157.

\section{REFERENCES}

[1] Rhodes, C.J., Anderson, R.M. (1996): A scaling analysis of measles epidemics in a small population. - Philos. Trans. R. Soc. Lond. B Biol. Sci. 351: 1679-1688.

[2] Rhodes, C.J., Anderson, R.M. (1996): Power laws governing epidemics in isolated populations. -- Nature 381: 600-602.

[3] Rhodes, C.J., Jensen, H.J., Anderson, R.M. (1997): On the critical behaviour of simple epidemics. - Proc. R. Soc. Lond. B Biol. Sci. 264: 1639-1646.

[4] Rhodes, C.J., Butler, A.R., Anderson, R.M. (1998): Epidemiology of communicable diseases in small population. - J. Mol. Med. 76: 111-116.

[5] Trottier, H., Philippe, P. (2005): Scaling properties of childhood infectious diseases epidemics before and after mass vaccination in Canada. - Journal of Theoretical Biology Biol. 235: 326-337.

[6] Philippe, P. (2000): Epidemiology and self-organized critical systems: An analysis in waiting times and diseases heterogeneity. - Nonlinear Dyn. Psychol. Life Sci. 4 (4): 275295.

[7] Harnos, A., Reiczigel, J., Solymosi, N., Vattay, G. (2006): Analysis of the Effect of Immunization in Rabies Time Series. - Journal of Theoretical Biology (in press)

[8] Mocsári, E., Kerekes, B., Heltay, I., Szalay, D., Csabay, L. (1994): Experiences on the oral vaccination of foxes against rabies in Hungary. - Hungarian Veterinary Journal 49: $10-15$.

[9] Rubel, F., Höflechner, A. (2004): Documentation of Rabies in Austria 1945-2003. Meeting of the Task Force Subgroup RABIES, Klagenfurt, Austria, 21 - 22 Sept. 2004.

[10] Institute for Internationa Cooperation in Animal Biologic An OIE Collaborating Center Iowa State University, College of Veterinary Medicine (2003): Bovine Tuberculosis 235: 326-337 (http://www.vm.iastate.edu/services/institutes/iicab/iicab.htm)

[11] Varga, J., Tuboly, S., Mészáros, J.(1999): A háziállatok fertőző betegségei. Állatorvosi járványtan II. Mezőgazda Kiadó

[12] Englehardt, J. D. (2002): Scale Invariance of Incident Size Distributons in Response to Sizes of Their Causes - Risk Analysis. 22: 2 